\title{
Güneş Enerji Sistemlerinde Sürekli Mıknatıslı DC Motorun Benzetimi ve 2 - DOF PI Kontrolörü ile Kontrolü
}

\author{
Kenan YANMAZ \\ Giresun Üniversitesi, Teknik Bilimler Meslek Yüksekokulu, Giresun, Türkiye
}

Sorumlu Yazar: kenan.yanmaz@giresun.edu.tr

Geliş Tarihi: 19.11 .2018 Kabul Tarihi: 27.11 .2018

$\ddot{\mathbf{O} z}$

Sürekli Mıknatıslı Doğru Akım (PMDC) Motorları hız kontrol uygulamalarında ve konum kontrolünün önemli olduğu durumlarda kullanılan gelişmiş elektrik motorlarıdır. Dolayısıyla bu motorların benzetimi ve kontrolü üzerine pek çok uygulama bulunmaktadır. Güneş enerji sistemleri günümüzde yaygın olarak kullanılan ve gelecekte kullanımı artacak olan bir yenilenebilir enerji kaynağıdır. Bu çalışmada güneş enerji sistemleri için PMDC motorun ayrıntılı matematiksel denklemlerinden yola çıkılarak benzetimi yapılmakta ve iki serbestlik dereceli PI (2-DOF PI) kontrolör devresi kullanılarak sistemin kontrolü gerçekleştirilmektedir. Benzetim çalışmaları MATLAB/Simulink ortamında gerçekleştirilmiş̧ir.

Anahtar Kelimeler: Sürekli mıknatıslı DC motor, PID, 2-DOF PI.

\section{Simulation and Control with $2-$ DOF PI Controller of Permanent Magnet DC Motors for Solar Energy Systems}

\begin{abstract}
Permanent Magnet Direct Current (PMDC) Motors are advanced electric machines used in speed control applications and where position control is important. Therefore, there are many applications on the simulation and control of these machines. Solar energy systems are a widely used today and is a renewable energy source that will increase its use in the future. In this study, the PMDC motor for solar energy systems is simulated based on detailed mathematical equations and the system is controlled by using two degree of freedom PI (2-DOF PI) controller circuit. Simulation studies were performed in MATLAB / Simulink environment.
\end{abstract}

Keywords: Permanent magnet DC motor, PID, 2-DOF PI. 


\section{Giriş}

Güneş enerjisi günlük hayatımızda hemen hemen her alanda kullanılmaktadır. Gerek su 1sıtmak için gerek elektrik enerjisi elde etmek için yaygın olarak kullanılmaktadır. Elektrik enerjisi üreten güneş panelleri fotovoltaik güneş panelleridir. Ev tipi uygulamalarda kullanılabildiği gibi büyük ölçekli uygulamalarda da kullanılmaktadır (Mengi, 2018).

PMDC motorlar endüstride yaygın olarak kullanılmaktadırlar. Klimalar, bilgisayarlar, tarla sulama, araba silecek sistemleri gibi birçok alanda kullanılmaktadır. PMDC motorları herhangi bir uyarma akımına ihtiyaç duymaz (Eminoğlu ve Altaş, 1998). Bu nedenle standart DC motorlara göre daha verimlidir. PMDC motorların sarım alanına gerek yoktur, bu nedenle PMDC motorların boyutu geleneksel DC motorlardan daha küçüktür ve PMDC motorların maliyeti nispeten düşüktür. Değişken hız gerektiğinde PMDC motor sürücüleri yüksek hızda çalışma için caziptir. Farklı şekillerde tasarlanabilirler ve geniş bir aralıkta yüksek verimlilik sergilerler (Bianchi ve ark., 2006). Yüksek performanslı DC motor sürücüler, PMDC motorları kullanır (Zenk, 2016) ve hızlı yanıt, küçük kararlı durum hatası, küçük aşma, hızlı yükselme süresi ve minimum oturma süresi ile hassas karmaşık konum-hız referans yörünge takibi gerektirir (Damiano ve ark., 1999). (Okonkwo, 2006) 'da, sistem yanıt hızını iyileştirmek için firçasız PMDC motor sürücü sistemi için bir nöral ağa dayanan sabit bir referans belirleme tekniği ve aşma ve salınımın azaltılması önerilmiştir.

Makine davranışını doğru şekilde temsil edebilen birçok DC motor modeli vardır, ancak modelin parametreleri doğru davranış sağlamak için matematiksel modelde doğru şekilde ayarlanmalıdır (Yıldız, 2012; Saab ve Kaed-Bey, 2001).

$\mathrm{Bu}$ çalışmada PMDC motorun devir sayısı Fractional - PI kontrol tekniği kullanılarak kontrol edilmektedir. Farklı referans seviyeleri denenerek kontrolörün etkinliği gösterilmiştir. Hazırlanan bu model ile sürülen motor değişik endüstriyel uygulamalarda kullanılabilecektir. Matlab/Simulink ortamında benzetimi yapılmıştır.

\section{Materyal ve Metot}

\section{1. Fotovoltaik Sistem}

Fotovoltaik güneş pilleri, güneş 1şığını elektrik enerjisine çeviren yarı iletken bileşenlerdir. Farklı eşdeğer devre modelleri vardır. Bir güneş panelinin bir diyotlu eşdeğer devre modeli Şekil 1'de görülmektedir (Altaş ve Sharaf, 2008). 


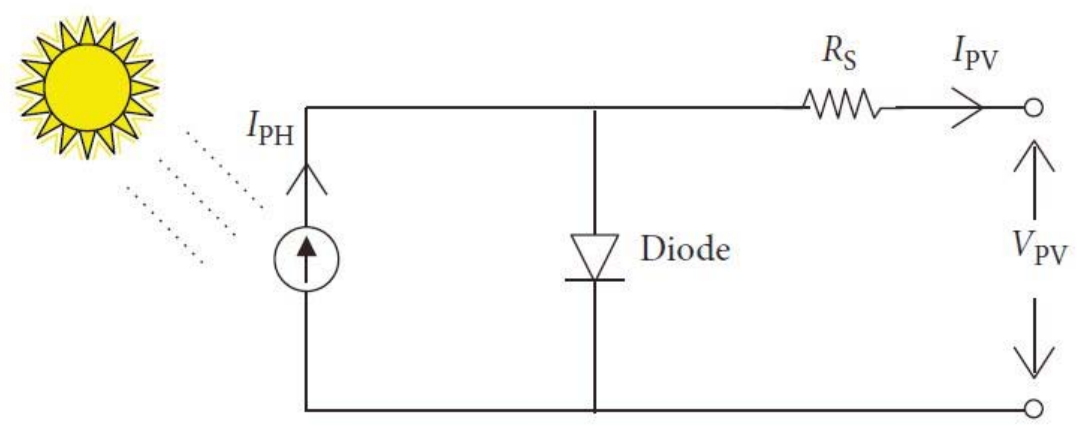

Şekil 1. PV güneş hücresinin eşdeğer devre modeli.

PV hücresinin çıkış gerilimi Denklem (1) 'de olduğu gibidir (Masoum ve ark., 2002).

$$
V_{P V}=\frac{N}{\lambda} \ln \left(\frac{I_{S C}-I_{P V}+M I_{0}}{M I_{0}}\right)-\frac{N}{M} R_{S} I_{P V}
$$

Burada, $V_{P V}$ PV hücresi çıkış gerilimini, $I_{P V}$ PV hücresi çıkış akımını, $I_{S C}$ PV hücresi kısa devre akımını, $I_{0}$ ters doyum akımını, $R_{S}$ PV hücresi seri direncini, $N$ seri hücre sayısını, $\lambda$ hücre malzemesine bağlı sabit katsayıyı, $M$ paralel hücre sayısını temsil etmektedir.

\section{2. PMDC Motor}

$\mathrm{Bu}$ bölümde PMDC motorlarının dinamikleri durum değişkenli bir denklem ile açıklanmaktadır. Matematiksel modeller, herhangi bir fiziksel sistemi anlamada temel öneme sahiptir. PMDC motor için dinamik bir model hem elektrik devresi hem de mekanik hareket denklemlerinden türetilmiştir. PMDC motorunun özelliklerini tanımlayan denklemler Denklem (2)'deki gibidir (Zenk and Akpınar, 2014).

$$
\left[\begin{array}{c}
\frac{d i_{a}}{d t} \\
\frac{d w_{m}}{d t}
\end{array}\right]=\left[\begin{array}{cc}
-\frac{R_{a}}{L_{a}} & -\frac{K_{m}}{L_{a}} \\
\frac{K_{T}}{J_{m}} & -\frac{B_{m}}{J_{m}}
\end{array}\right]\left[\begin{array}{c}
i_{a} \\
w_{m}
\end{array}\right]+\left[\begin{array}{c}
\frac{V_{a}}{L_{a}} \\
-\frac{T_{L}}{J_{m}}
\end{array}\right]
$$

Burada $i_{a}$ motor akımını, $w_{m}$ rotor hızını, $T_{L}$ yük torkunu, $B_{m}$ sürtünme sabitini, $R_{a}$ armatür direncini, $J_{m}$ rotorun ataletini, $L_{a}$ armatür endüktansını, $K_{T}$ zıt elektromotor kuvvet veya tork sabitini, $K_{m}$ gerilim sabitini, $V_{a}$ armatür gerilimini temsil etmektedir. 
PMDC motorda, elektromanyetik tork $\left(T_{E}\right)$ ve zit elektromotor kuvvet, sirasiyla motor akımı ve hız motoru ile orantılıdır. Zit elektromotor kuvvet $\left(K_{T}\right)$ mıknatısın gücü, demirin direnci ve armatür sargısının dönüş sayısı ile belirlenir. Stator manyetik akısı, tüm armatür akımı seviyelerinde esas olarak sabit kalır, bu nedenle PMDC motorunun tork-hız eğrisi doğrusaldır.

Motorun transfer fonksiyonu, sistemin yüksek performansını iyileştirecek uygun kontrol cihazını tasarlamak için önemlidir.

PMDC motoru, sadece taban hızının altındaki motor hız kontrolünün gerekli olduğu ve elektronik yöntemlerle armatür voltaj kontrolü ile elde edildiği sistemlerde bulunabilir. Lineer ve lineer olmayan kontrol sistemlerinde en kullanışlı kontrol algoritmalarından biri PID kontrolüdür. PID kontrol cihazı, sadeliği ve sağlamlığı nedeniyle endüstride yaygın olarak kullanılmaktadır. Motor hızı, hata hızını oluşturmak için referans hız ile karşılaştırılır. PID kontrol cihazının transfer fonksiyonu Denklem (3)'te verilmektedir.

$$
G_{k}(s)=K_{P}+\frac{K_{I}}{s}+K_{D} s
$$

Burada, $K_{P}$ sabit kazanc1, $K_{I}$ integral kazancını ve $K_{D}$ ise türevsel kazancı ifade etmektedir.

\section{3. 2 - DOF PI Kontrolör}

Mevcut endüstriyel kontrol uygulamalarının çoğunda olduğu gibi, kontrol edilen değişken veya ayar noktasının istenen değeri normalde sabit kalır ve değiştirilmesi gerekir. Bu kontrol tekniği esas olarak PID kontrol algoritmalarının iki serbest dereceli (2-DOF) uygulaması ile ilgilenmektedir. 2DOF kontrol algoritmasının sağladığı ek parametre, regülatif kontrol performansı ve kapalı döngü kontrol sistemi dayanıklılığı göz önüne alındığında servo kontrol davranışlarını iyileştirmek için kullanılır [1-5]. Bu 2-DOF özelliği hem bir PI'ye hem de PID kontrol algoritmasına dahil edilebilir.

2-DOF kontrolör yapısı bir serbestlik dereceli (1-DOF) kontrolör yapısının yetersiz kaldığı durumlar için geliştirilmiştir. Kontrolör yapısına $\beta$ parametresinin eklenmesiyle referans bozucu bastırma performansını koruma açısından ve geçici durum cevabı iyileştirilmesi açısından daha iyi performans sağlanmaktadır. 2-DOF PID kontrolör yapısı Şekil 2'de görülmektedir. 


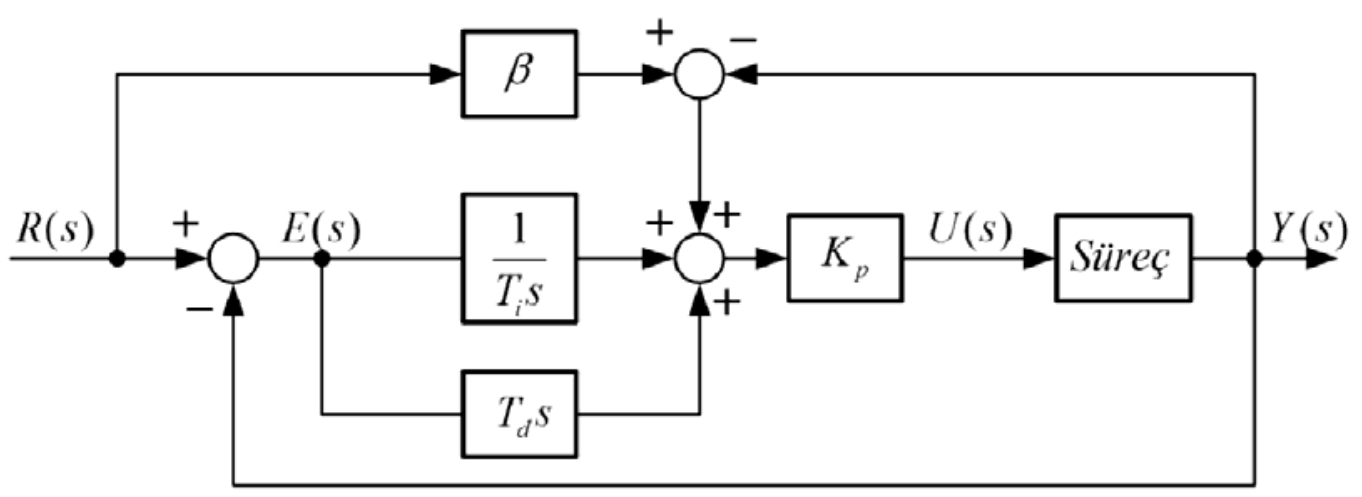

Şekil 2. 2-DOF PID yapısı.

2-DOF PI kontrolör için kontrol işareti Denklem (4)’te olduğu gibidir.

$$
\begin{aligned}
& u(s)=K_{P}\left(\beta+\frac{1}{T_{i} S}\right) r(s)-K_{P}\left(1+\frac{1}{T_{i} S}\right) y(s) \\
& u(s)=C_{r}(s) r(s)-C_{y}(s) y(s)
\end{aligned}
$$

Burada $T_{i}$ integral zaman sabitini, $K_{P}$ oransal kazancı, $\beta$ referans ağırlık oranını, $C_{r}(s)$ referans işaret transfer fonksiyonunu ve $C_{y}(s)$ geri besleme transfer fonksiyonunu göstermektedir (Alfaro ve Vilanova, 2016).

\section{Bulgular ve Tartışma}

Benzetimi yapılan sistemdeki motor parametreleri Tablo 1'de verilmektedir (Shahgholian ve Shafaghi, 2010).

Tablo 1. DC motor parametreleri.

\begin{tabular}{cc}
\hline Parametre & Değer \\
\hline Armatür direnci $\left(R_{a}\right)$ & $1.4 \Omega$ \\
Armatür endüktans1 $\left(L_{a}\right)$ & $0.0805 \mathrm{H}$ \\
Gerilim sabiti $\left(K_{m}\right)$ & $0.095 \mathrm{~V} / \mathrm{rad}$ \\
Tork sabiti $\left(K_{T}\right)$ & $0.095 \mathrm{Nm} / \mathrm{A}$ \\
Rotorun ataletini $\left(J_{m}\right)$ & $0.0007432 \mathrm{kgm}$ \\
Sürtünme sabiti $\left(B_{m}\right)$ & $0.000431 \mathrm{Vs} / \mathrm{rad}$ \\
Armatür gerilimi $\left(V_{a}\right)$ & $36 \mathrm{~V}$ \\
\hline
\end{tabular}

Benzetimi yapılan sistemin Matlab/Simulink modeli Şekil 3’te görülmektedir. 


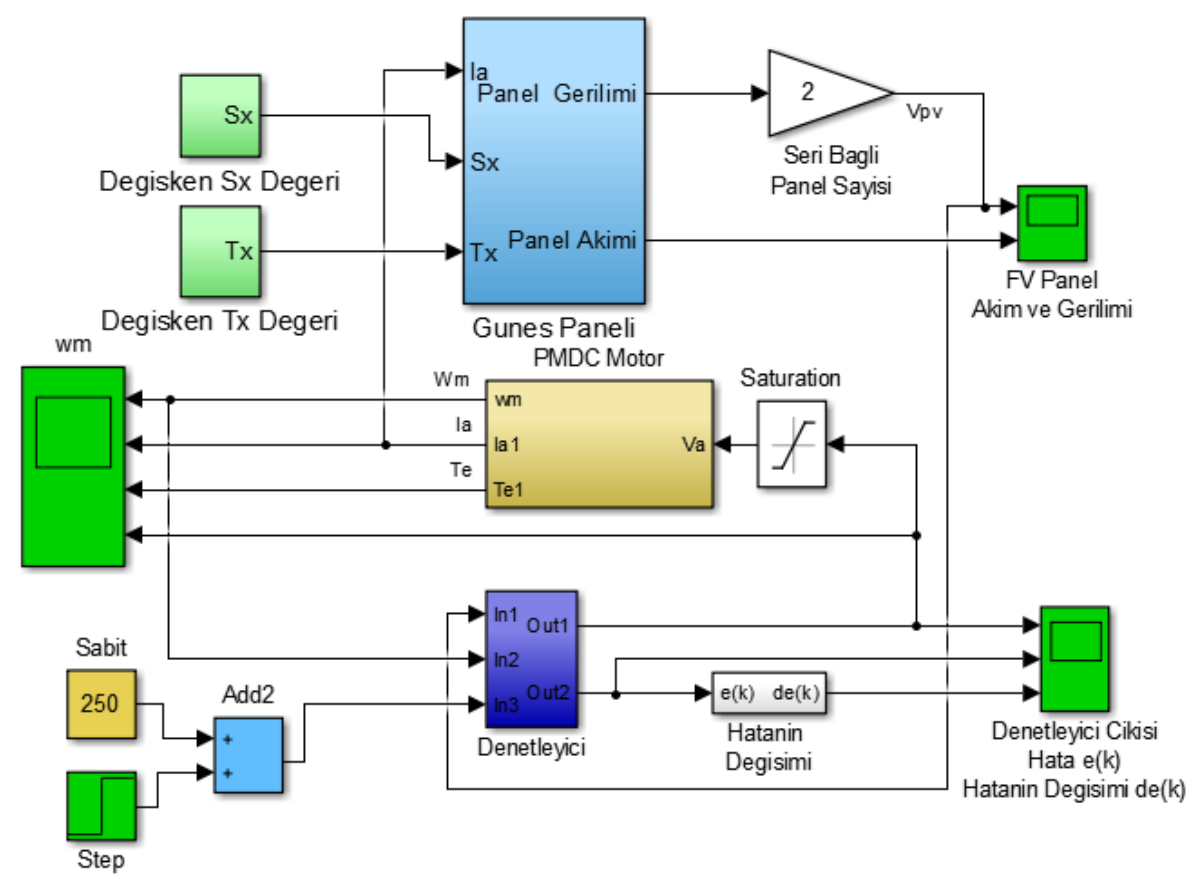

Şekil 3. Benzetimi yapılan sistemin Matlab/Simulink modeli.

Kullanılan denetleyici yapısında $K_{P}=0.5534, T_{i}=2.6728$ ve $\beta=1.1$ değerleri alınmıştır. Şekil 4'te motor hızının referans değer ile karşılaştırılması verilmiştir. Referans değer ikinci saniyede 250 değerinden 300 değerine çıkarılmaktadır. Şekilden de görüldüğü gibi bu sistem için tasarlanan kontrol devresi ile motor çıkış hız değerinin referans değeri başarılı bir şekilde izlediği net bir şekilde görülmektedir.

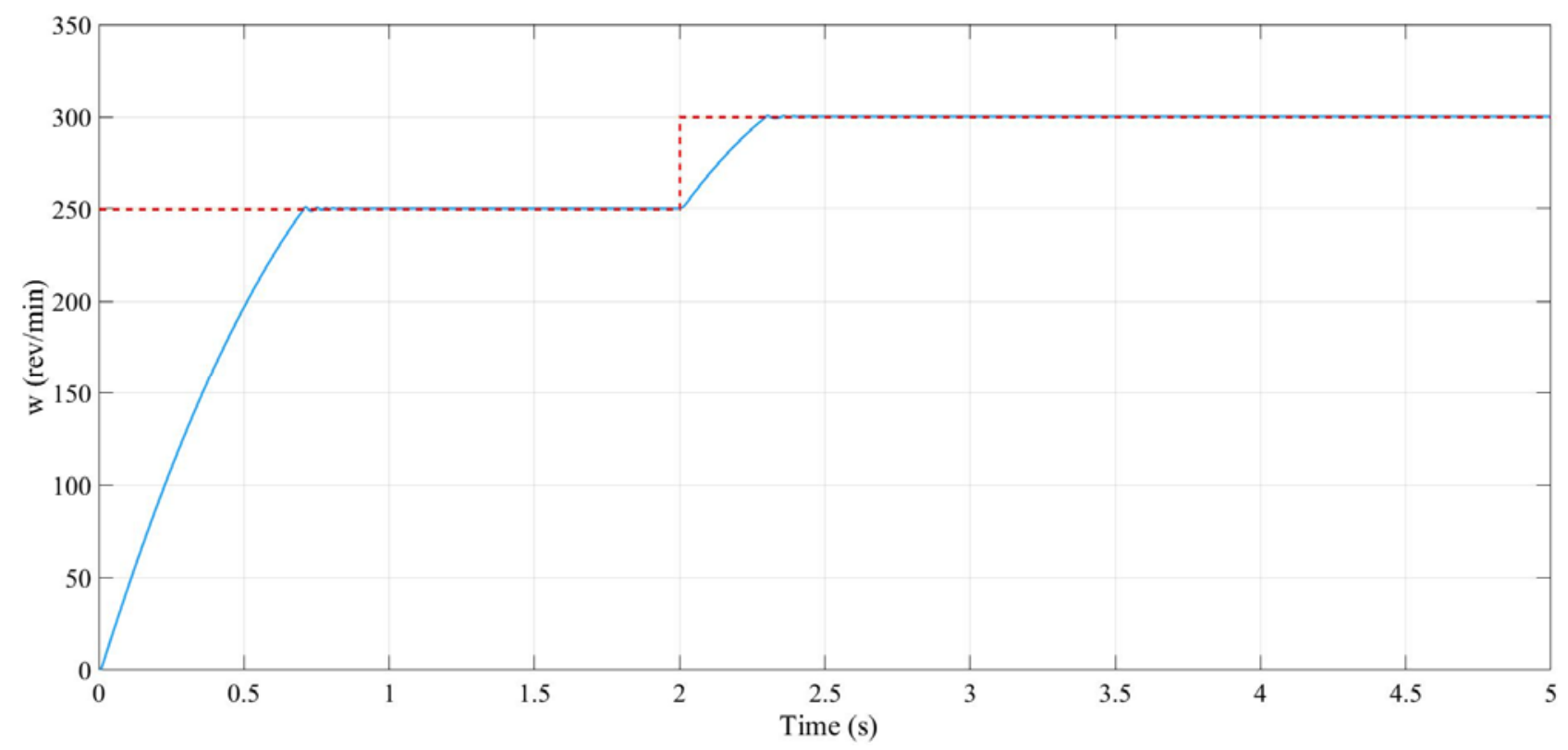

Şekil 4. Motor hızı ve referans değerleri değişimi. 
Şekil 5'te kontrolör çıkışındaki işaretin dalga şekli, kontrol edilen hız ile referans değer arasındaki farkın oluşturduğu hata değeri ve hatadaki değişim grafikleri görülmektedir.
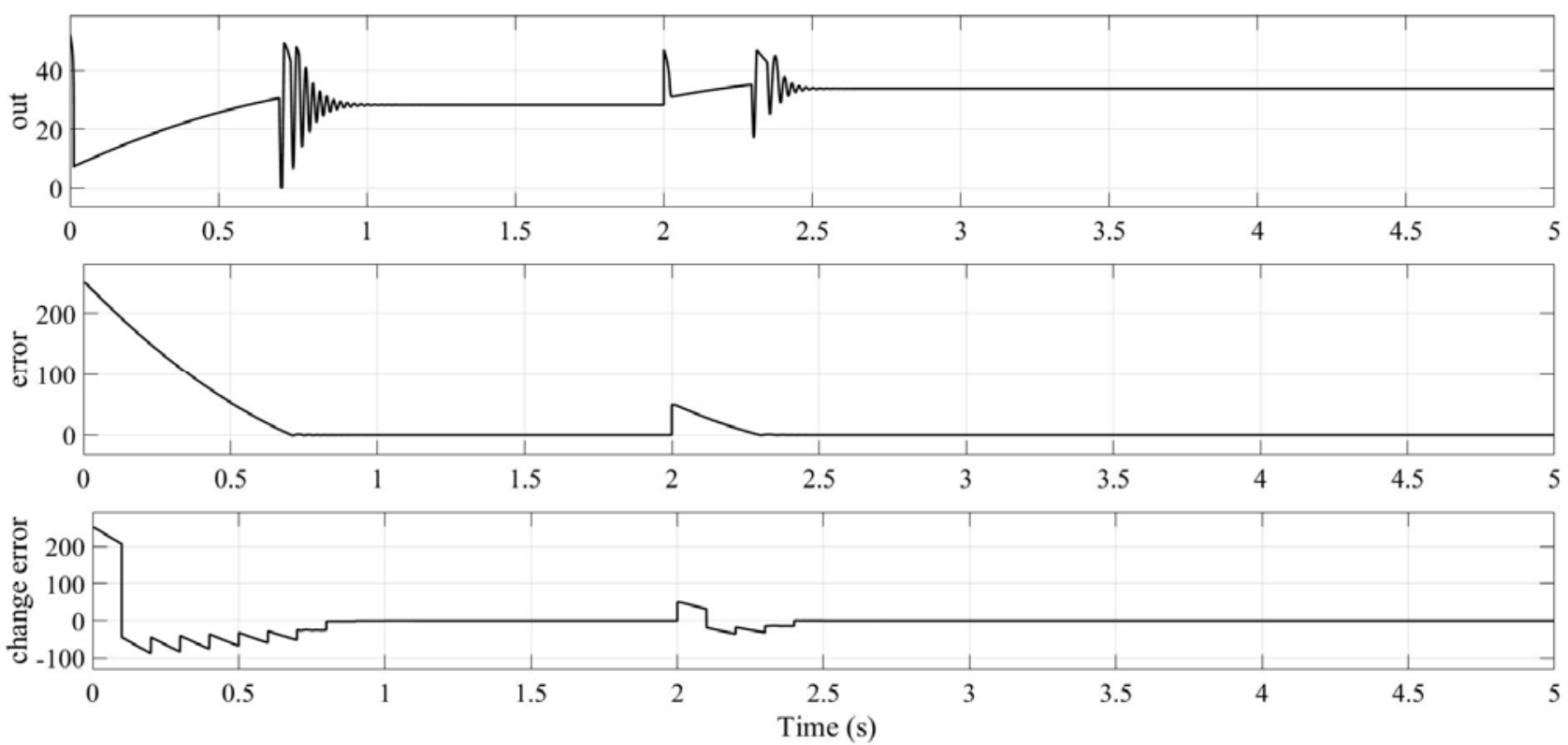

Şekil 5. Kontrolör çıkışı, hata ve hatadaki değişim.

\section{Sonuçlar ve Öneriler}

Sürekli mıknatıslı doğru akım motorları hız kontrol uygulamalarında ve konum kontrolünün önemli olduğu durumlarda yaygın bir kullanım alanına sahiptir. Güneş enerji sistemleri günümüzde yaygın olarak kullanılan ve gelecekte kullanımı artacak olan bir yenilenebilir enerji kaynağıdır. Çeşitli amaçlar için kullanılabilecek olan, güneş pilleri ile birlikte kullanılan PMDC motor devresi hazırlanmış ve MATLAB/Simulink ortamında benzetimi yapılmıştır. PMDC motorun ayrıntılı matematiksel denklemlerinden yola çıkılarak benzetimi yapılmış ve iki serbestlik dereceli PI kontrolör devresi kullanılarak sistemin kontrolü gerçekleştirilmektedir. Elde edilen sonuçlar bu kontrol sisteminin bu tip uygulamalar için kullanışlı ve bu gibi uygulamalar için daha da geliştirilebilir olduğunu ortaya koymaktadır.

\section{Kaynaklar}

Alfaro, V. M., ve Vilanova, R., (2016). Model Reference Robust Tuning of PID Controllers. Springer International Publishing.

Altaş, I. H., ve Sharaf, A. M. (2008). A novel maximum power fuzzy logic controller for photovoltaic solar energy systems. Renewable Energy, 33(3), 388-399.

Bianchi, N., Bolognani, S., ve Luise, F., (2006). High speed drive using a slotless PM motor. IEEE Tran. On Pow. Elec., 21(4), 1083-1090. 
Damiano, A., Gatto, G., Pisano, A., ve Usai, E., (1999). Digital second order sliding mode control of PMDC motor. IEEE International Symposium on Industrial Electronics (ISIE’99), Slovenia, 1, 322-329.

Eminoğlu, İ., ve Altaş, İ. H., (1998). The effects of the number of rules on the output of a fuzzy logic controller employed to a PM d.c. motors. Computers and Electrical Engineering, 24(1998), 245-261.

Masoum, M. A. S., Dehbonei, H., ve Fuchs, E. F., (2002). Theoretical and experimental analyses of photovoltaic systems with voltage and current-based maximum power-point tracking. IEEE Transactions on Energy Conversion, 17(4), 514-522.

Mengi, O. Ö. (2018). A Five-Level H-Bridge STATCOM for an Off-Grid PV Solar Farm under Two Controllers PI and $\mathrm{PI}^{\lambda}$-MPC Hybrid. International Journal of Photoenergy, 2018, 1-14.

Okonkwo, R. C., (2006). Design and performance of permanent magnet DC linear motors. IEEE Trans. On Magn., 42(9), 2179-2183.

Saab, S. S., ve Kaed-Bey, R. A., (2001). Parameter Identification of a DC Motor: An Experimental Approach. IEEE International Conf. on Elec. Circuit and Systems. (ICECS '01), 4, 981-984.

Shahgholian, G., ve Shafaghi, P., (2010). State Space Modeling and Eigenvalue Analysis of the Permanent Magnet DC Motor Drive System. 2nd International Conference on Electronic Computer Technology, 7-10 May 2010, Kuala Lumpur, Malaysia, 63-67.

Yildiz, A. B., (2012). Electrical equivalent circuit based modeling and analysis of direct current motors. Electrical Power and Energy Systems, 4, 1043-1047.

Zenk, H., (2016). A Comparative Application of Performance of the SEPIC Converter Using PI, PID and Fuzzy Logic Controllers for PMDC Motor Speed Analysis. Journal of Multidisciplinary Engineering Science Studies (JMESS), 2(12), 1226-1231.

Zenk, H., Akpinar, A., S., (2014). Dynamic Performance Comparison of Cúk Converter with DC Motor Driving and Using PI, PID, Fuzzy Logic Types Controllers. Universal Journal of Electrical and Electronic Engineering, 2 , 90 - 96. doi: 10.13189/ujeee.2014.020206. 